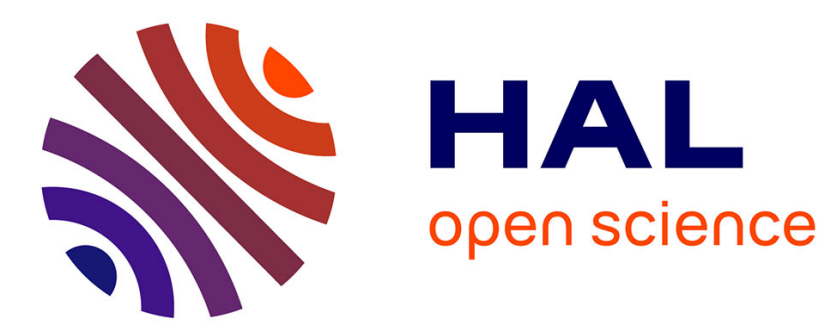

\title{
Design and Measurements of a Quasi-isotropic UWB Planar Antenna
}

Antoine Diet, Nicolas Ribière-Tharaud

\section{To cite this version:}

Antoine Diet, Nicolas Ribière-Tharaud. Design and Measurements of a Quasi-isotropic UWB Planar Antenna. Recent patents on Electrical Engineering, 2011, 4 (3), pp.176 -183. 10.2174/1874476111104030176 . hal-00641208

\section{HAL Id: hal-00641208}

https://hal-centralesupelec.archives-ouvertes.fr/hal-00641208

Submitted on 17 Nov 2011

HAL is a multi-disciplinary open access archive for the deposit and dissemination of scientific research documents, whether they are published or not. The documents may come from teaching and research institutions in France or abroad, or from public or private research centers.
L'archive ouverte pluridisciplinaire HAL, est destinée au dépôt et à la diffusion de documents scientifiques de niveau recherche, publiés ou non, émanant des établissements d'enseignement et de recherche français ou étrangers, des laboratoires publics ou privés. 


\title{
Design and Measurements of a quasi-isotropic UWB micro-strip antenna
}

\author{
Antoine Diet, Nicolas Ribière-Tharaud \\ Laboratoire des Signaux et Systèmes / Département de Recherche en Électromagnétisme \\ L2S/DRÉ UMR 8506 (CNRS-Supélec-Université Paris-Sud-11) \\ Supélec, 3 rue Joliot Curie, plateau du Moulon F-91192 Gif sur Yvette, France \\ antoine.diet@lss.supelec.fr
}

\begin{abstract}
This paper summarizes the design and measurements of a quasi-isotropic printed UWB antenna, with the analysis of its performances through defined figures of merit. State of the art gives the opportunity to exploit some empirical considerations about the shape and the methodology of conception. A simple structure is simulated by means of CST Microwave Studio to show the possibility of fulfilling, at a defined level, UWB antenna requirements such as matching, fidelity and quasi-isotropy of the radiation pattern with a simple micro-strip design and a standard technology. The design of this antenna is based on considerations about bulbous and bended traveling wave antennas. Results of its characterization in anechoic chamber are quantified in terms of amplitude and phase variation, in order to illustrate the level of dispersion for the different directions.
\end{abstract}

\section{I - INTRODUCTION ABOUT UWB ANTENNAS}

In the context of wireless local and mobile networks, UWB communications systems are an interesting solution to avoid the problem of standards co-existence and interferences [1]. The immunity of such systems is based on the wideband and low power pulses transmission. We focus herein on UWB communication systems that are based upon $3-11 \mathrm{GHz}$ antennas. The goal is to transmit very low power pulses in order to achieve sufficient data rate without disturbing other neighboring wireless communications that share part of the UWB band. Antennas dedicated to such applications are not supposed to be multi-harmonic but they are required to be really non-dispersive and wideband. Power matching should be realized continuously on the whole $7.5 \mathrm{GHz}$ wide frequency band. Furthermore, the phase transfer response must be linear with respect to frequency and ideally for any direction of emission, as mobility considerations require UWB antennas to be as isotropic as possible. Both frequency and time domain responses must be considered during the design. The dispersion that can be observed on radiated pulses reveals the antenna time behavior. Integration and production of UWB mobile transceivers imply an additional task that consists in building a minimum-size low-cost antenna. These considerations lead to avoid volumetric structures (i.e., biconical, 3D monopoles or horns). However studying the latter as possible UWB antennas is essential to find design rules for other technologies. Micro-strip antenna shapes, for example, are often inspired by projection of $3 \mathrm{D}$ existing antennas. In short, integration of an UWB antenna requires the latter to be isotropic (almost) and matched over a wide band and to show a minimum size, a low cost and a low dispersion (continuously). UWB antenna property, in their fundamental idea, provides a variation in amplitude in phase that should not destroy a signal using the whole bandwidth continuously: a pulse. This is pointed out because lots of wideband antenna are in fact multi-narrowband ones. As a consequence, the difference between wideband and impulse wideband property lie in the time domain analysis.

Designing this type of antenna is not a recent challenge and this is proved by the important state of the art (biconical, horn...) [2]-to-[15]. Interest is in the time domain approach and the new technical considerations such as integrated antenna and no more 3D structures. The goal is to reduce the size for a potential integration of an UWB communication system for mobility and connectivity applications. We focused on printed bulbous traveling waves antennas, in order to avoid reflexion and multi-resonant structure. A wideband resonating solution would drive it to a crippling effect of ringing, generating time dispersion of the pulse in the time domain unless the antenna is matched. We favored the quasi isotropic and dual polarization property by reducing the ground plane (this increases the sensibility also). This was justified in the way that this antenna is supposed to be used as an energy detector. A potential application of such an antenna can be the transmission of BAN (Body Area Network) signals, in the context of nomadic sensors networks.

\section{II - STATE OF THE ART AND FIGURES OF MERIT}

As reported by Schantz [10], the interest in wideband antennas is old. History begins with biconical 3D antennas. It goes on with horns and spherical fat structures with interesting wideband properties [10]. In 1940, Kings claims that "fatter is better", arguing that the stored reactive energy is reduced in bulbous antenna [2, 3]. Then, a lot of work has been done on volumetric curve based or tapered antenna. Frequency independent antennas, based only on angles, were studied by Rumsey [12]. This was the introduction of spiral and log-periodic antennas, although the latter are dispersive due to phase centre translation in frequency. Nowadays, modern UWB antennas have to account for several considerations: matching properties, minimum reactive power 
and resonance (which leads to thick or bulbous structures), low cost and small size (which lead to printed antennas). Several technologies can be used to realize wideband antennas [1][5][10][8]. Many authors consider planar monopoles and slot line antennas [5, 9]. The most important constraint is to build a non-resonant structure, which generally implies wide bent surfaces of conductor, printed on substrates. Traveling wave structures are also preferred because they are not subject to geometric resonances. This causes the success of the Vivaldi antenna [8] despite its directional radiation pattern. Considering practical realization requirements (size and cost), the printed antenna is very popular for UWB. The geometry is first designed for a wide radiation pattern and, then, optimized for frequency matching. Figure 1 displays two configurations summarized in [8]: The ring tapered slot and the horn type printed antenna. These solutions offer good matching and radiation patterns for several tens of square millimeters. References [5] and [6] present slot antennas and monopoles that are matched in a second step by a modification of the geometric parameters. It seems that thick printed structures offer the wideband behavior necessary for UWB in a first step, bent shapes being chosen to radiate with large beam-width and ground plane being avoided to enable isotropic radiation. The second step is to consider both matching and dispersion. These empirical considerations have been accounted for in the simulation of the bent elliptical monopole described in the next part.
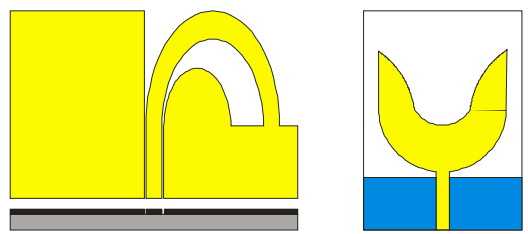

Figure 1 : Half-ring tapered slot (left) and horn type (right) printed UWB antennas reported in [8]

In order to analyze the results in the frequency and time domains, we use different figures of merit. Matching is characterized by means of return loss (RL) calculation as a function of the frequency: $\left|S_{11}\right|(f)$ in $\mathrm{dB}$. For the radiation pattern (RP), the observation in a $2 \mathrm{D}$ projection enables us to identify gaps (whose locations, in spherical coordinates, are indicated by limits in $\theta$ and $\varphi$ ) that severely impact on the antenna isotropic properties. We propose to characterize isotropy by means of an "isotropic" factor $I$ defined at one frequency and for a reference level $x$ (expressed in $\mathrm{dBi}$ ). The factor $I$ is as follows:

$$
\begin{aligned}
& I_{x\left[d B_{i}\right]}=\sum_{n}^{\substack{N \text { Radiation } \\
\text { Pattern Areas }}} I_{n}^{x} \\
& I_{x}^{n}=\left|\frac{1}{4 \pi} \int_{\theta_{\min (x)}^{n}}^{\theta_{\max (x)^{n}}^{\varphi_{\max }^{n}(x)}} \int^{\varphi_{\min }^{n}(x)} \cos (\varphi) \mathrm{d} \theta \mathrm{d} \varphi\right|
\end{aligned}
$$

$$
I_{x}^{n}=\frac{\left|\theta_{\max (x)}^{n}-\theta_{\min (x)}^{n} \| \sin \left(\varphi_{\min (x)}^{n}\right)-\sin \left(\varphi_{\max (x)}^{n}\right)\right|}{4 \pi}
$$

The lower the factor $I_{x}$ for a given level $x$, the better is our antenna for isotropy considerations. In fact, this factor corresponds to the area (expressed in str.) where the radiated power level is less than $x \mathrm{dBi}$, normalized with respect to the area corresponding to a reference isotropic antenna radiating $x \mathrm{dBi}$, i.e., $4 \pi$ str.

For time domain analysis, we use the definition of fidelity, derived from [9] and expressed by equation (4). As it is clearly understood, the fidelity quantifies the time pulse correlation, for one direction of radiation, compared to the ideal one, i.e., the feeding signal (often a Gaussian pulse, or one of its derivatives).

$$
\mathrm{F}(\theta, \varphi)=\left[\frac{\mathrm{R}_{\mathrm{xy}}(\tau)}{\sqrt{\mathrm{R}_{\mathrm{xx}}(0) \mathrm{R}_{\mathrm{yy}}(0)}}\right]_{\max (\tau)} \quad \text { with }\left\{\begin{array}{l}
\mathrm{x}: \text { ideal pulse } \\
\mathrm{y}: \text { radiated pulse at }(\theta, \varphi)
\end{array}\right\}
$$

These three figures of merit: $20 . \log _{10}\left|S_{11}\right|, \quad I_{x}(f)$ and $F(\theta, \varphi)$, will help antenna designers to qualify UWB antenna simulations or measurements. As expressed in [11], an UWB antenna must be based on several considerations: wide bandwidth, fixed phase-centre over frequency, high radiation efficiency, low cost and small size. Other properties such as polarization and feeding techniques are considered in function of the transmission channels.

\section{III - SIMULATIONS OF A SIMPLE PRINTED STRUCTURE}

\section{A. Antenna description}

The structure is a bent micro-strip elliptical monopole, simulated under CST microwave studio. Its dimensions are $80 \mathrm{~mm} \times 60 \mathrm{~mm}$ and the substrate is a Rogers RT5880 $\left(\varepsilon_{r}=2.2\right)$ with a $1.575 \mathrm{~mm}$ thickness and a $70 \mu \mathrm{m}$ thick copper conductor (that corresponds to standard realization values). The feed is done by means of a matched micro-strip line with taper, the width varying for matching optimization. It is supposed to be directly connected to a $50 \Omega$ line or a $\mathrm{HF}$ connector. The design and photography is depicted in figure 2. The idea is to favor current flowing on a wide area and the isotropy is supposed to be influenced by the bending of the ellipse. The ellipse eccentricity and circular taper size were firstly optimized for matching on the whole bandwidth $\left(10 . \log _{10}\left|S_{11}\right|<-10 \mathrm{~dB}\right)$. In a second step the curvature was increase to generate à radiating field in different direction of the space. The angle was stopped at $180^{\circ}$ to avoid an annular resonating phenomenon that would have caused a ringing effect, source of time dispersion. The limitation of the ground plane enables the radiation in a «more » isotropic manner, and the resulting polarization is circular due to the current flowing. 


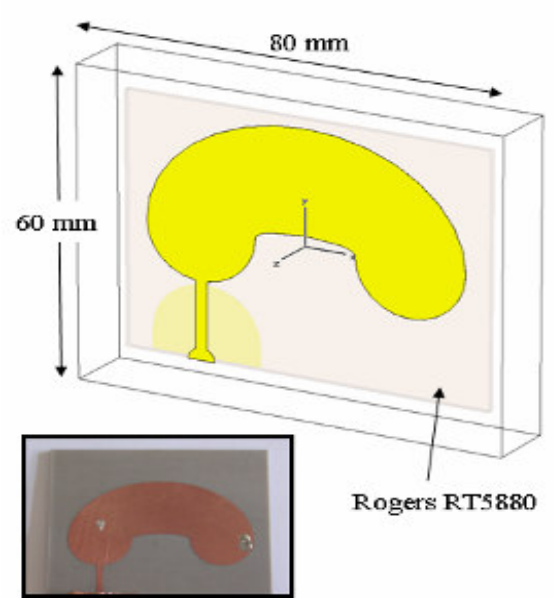

Figure 2 : Simulation and printed antenna designed

\section{B. Results of the simulation: return loss}

The first design criterion is to adapt the structure to $50 \Omega$ on the $3-11 \mathrm{GHz}$ band. Figures 3 and 4 display the final result, after a geometrical optimization. Figure 3 displays the behavior of the $S_{11}$ parameter versus the frequency; the adapted $50 \Omega$ point can be clearly seen at the centre of the trajectory. Figure 5 shows the return loss value: it is below $10 \mathrm{~dB}$, which corresponds to a classical limit for antenna adaptation.

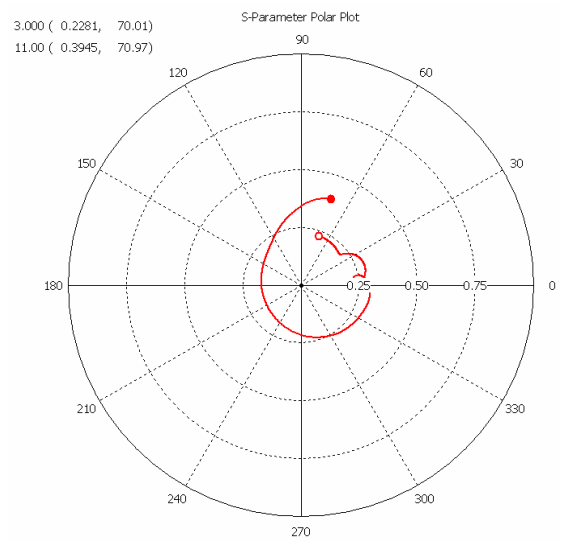

Figure $3: S_{11}$ parameter polar plot from 3 to $11 \mathrm{GHz}$

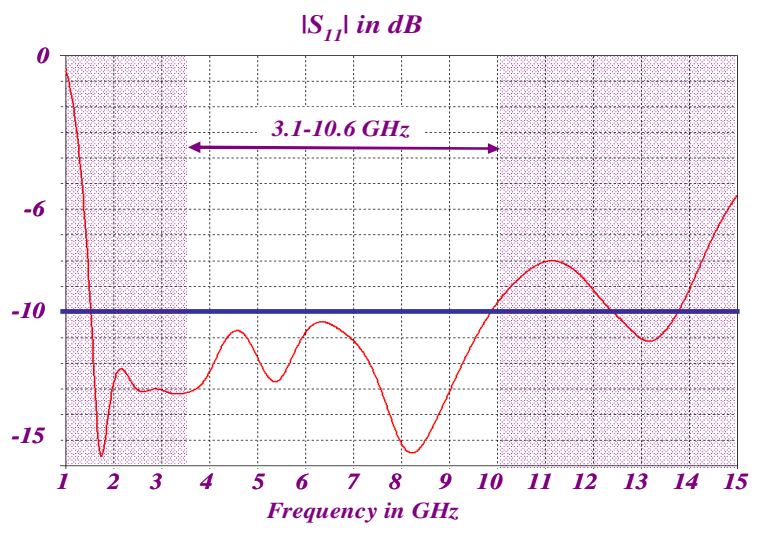

Figure 4: $\left|\mathbf{S}_{11}\right|$ antenna parameter

\section{Results of the simulation: radiation patterns}

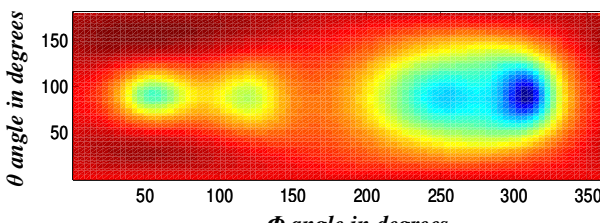

$4 \mathrm{GHz}$
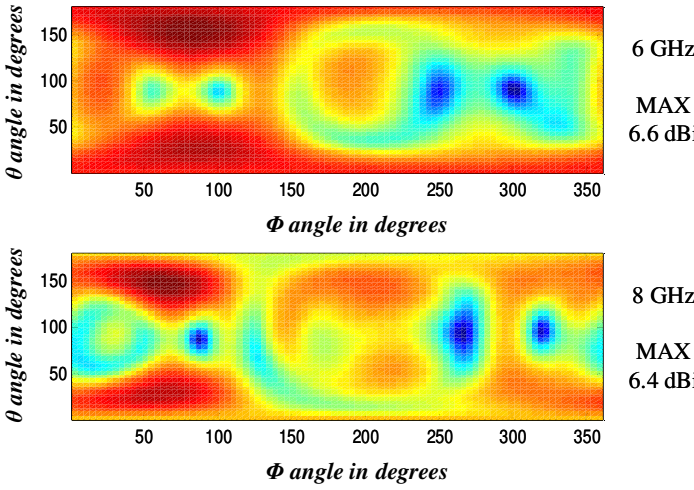

$8 \mathrm{GH}$

MAX $6.4 \mathrm{dBi}$

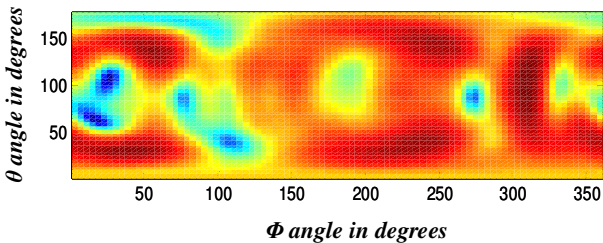

$10 \mathrm{GHz}$

MAX

$4.1 \mathrm{dBi}$

Figure $5: 2 \mathrm{D}$ maps of the radiation pattern at $4,6,8$ and $10 \mathrm{GHz}$ : the scale covers from $-30 \mathrm{~dB}$ to $0 \mathrm{~dB}$ with respect to the maximum value indicated on the right.

Radiation patterns at $4,6,8$ and $10 \mathrm{GHz}$ are reported in figure 5: they are displayed in the $(\theta, \varphi)$ plane corresponding to the angles used in equations (1), (2) and (3). This Figure illustrates antenna directivity. Some holes appear in directions $\left(\theta=90^{\circ}, \varphi=250^{\circ}-300^{\circ}\right)$ and $\left(\theta=90^{\circ}, \varphi=20^{\circ}-50^{\circ}\right)$. The radiation pattern is very omni-directional: the radiated field shows a gain near 0 dBi over a very large part of the space. This can be confirmed by computing the isotropy factor $I$ at different levels of gain.

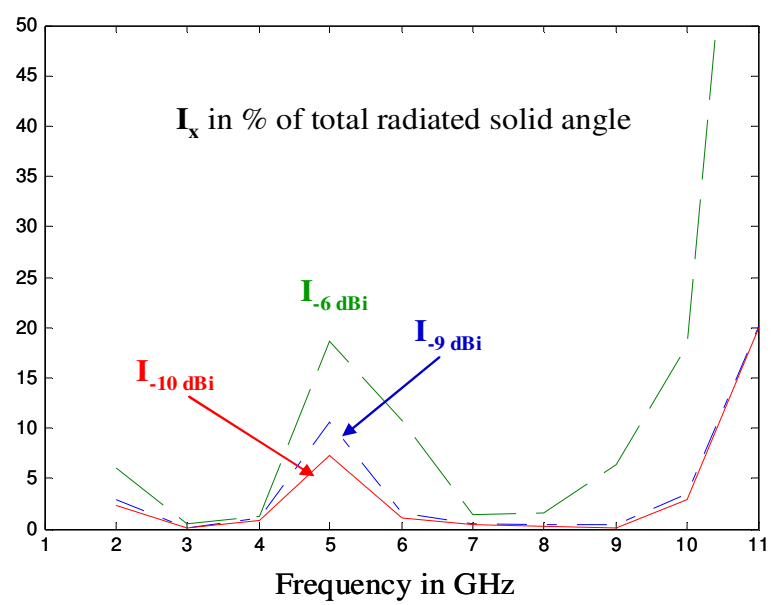

Figure 6: $I_{x}$ as a function of the frequency $(x=-6,-9,-10 \mathrm{dBi})$ 
Figure 6 displays the factor $I$ (at $x=-6,-9$ and $-10 \mathrm{dBi}$ ) as a function of the frequency. For example, at a level $x$ of $9 \mathrm{dBi}$, the area where the radiated power is less than $P_{\text {rad }}-9 \mathrm{~dB}$ represents less than $12 \%$ of the total area (where $P_{\text {rad }}$ is the power radiated by an ideal isotropic source). The behavior of the antenna radiation pattern reveals selectivity near 5_GHz; elsewhere the antenna radiates power in almost all directions. The quasi-circular nature of the polarization is depicted in figure 7 , which also displays the right and left polarization radiation patterns.

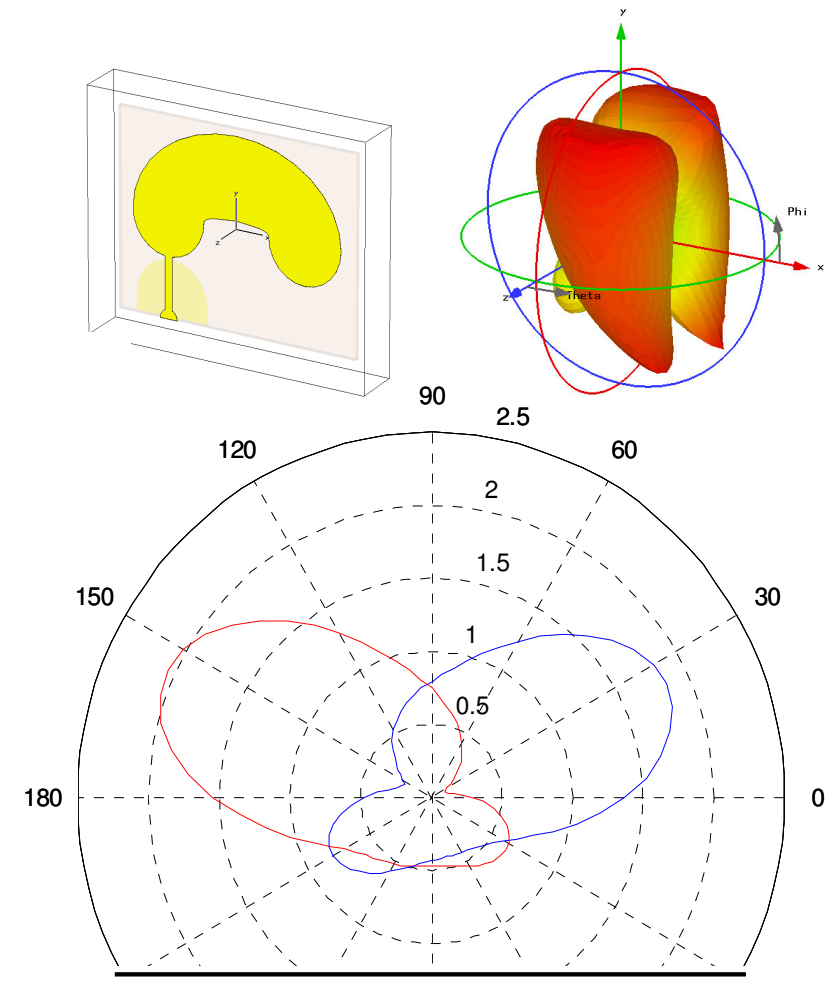

Figure 7: Antenna (top left) and axial ratio of the radiation pattern at $4 \mathrm{GHz}$ (top right). Lower row: left (blue) and right (red) polarisation radiation patterns as functions of $\theta\left(\varphi=0^{\circ}, 4 \mathrm{GHz}\right)$.

\section{Results of the simulation: Fidelity}

The fidelity is computed by simulating the response of linear probes, at a distance of 1 meter and for different positions. Spherical coordinates are used in Table 1, as in the paper. At each position, observation is performed by means of two linearly polarized probes oriented in the directions of the vectors $\theta$ and $\varphi$ respectively. It can be noticed that only three directions show fidelity lower than $71 \%$. For the latter, the fidelity computed at the same position but for the other orientation is almost $80 \%$. This means that it is always possible to improve the fidelity thanks to the orientation of probes at these locations. In figure 8 , the best case for the fidelity is obtained with a shift of $180^{\circ}$, which is the reason why the pulse appears as inverted. This is not damaging for a correlation receiver.
Table 1: Location, orientation and fidelity results of probes

\begin{tabular}{|c|c|c|c|}
\hline \multirow{2}{*}{\multicolumn{2}{|c|}{$\begin{array}{l}\text { Position angles in degrees } \\
\quad(\text { radius }=1 \mathrm{~m})\end{array}$}} & \multirow{3}{*}{$\begin{array}{l}\text { Orientation } \\
\text { vector }\end{array}$} & \multirow{3}{*}{$\begin{array}{c}\text { Fidelity to } \\
\text { emitted pulse }\end{array}$} \\
\hline & & & \\
\hline Théta $(\theta)$ & $\operatorname{Phi}(\varphi)$ & & \\
\hline $45^{\circ}$ & $0^{\circ}$ & $\theta$ & $76.2 \%$ \\
\hline $45^{\circ}$ & $0^{\circ}$ & $\varphi$ & $87.3 \%$ \\
\hline $130^{\circ}$ & $45^{\circ}$ & $\theta$ & $90.5 \%$ \\
\hline $130^{\circ}$ & $45^{\circ}$ & $\varphi$ & $96.2 \%$ \\
\hline$-130^{\circ}$ & $45^{\circ}$ & $\theta$ & $85.6 \%$ \\
\hline$-130^{\circ}$ & $45^{\circ}$ & $\varphi$ & $79.8 \%$ \\
\hline $130^{\circ}$ & $-45^{\circ}$ & $\theta$ & $83.7 \%$ \\
\hline $130^{\circ}$ & $-45^{\circ}$ & $\varphi$ & $69.2 \%$ \\
\hline $130^{\circ}$ & $130^{\circ}$ & $\theta$ & $85.4 \%$ \\
\hline $130^{\circ}$ & $130^{\circ}$ & $\varphi$ & $94.1 \%$ \\
\hline $45^{\circ}$ & $130^{\circ}$ & $\theta$ & $82.8 \%$ \\
\hline $45^{\circ}$ & $130^{\circ}$ & $\varphi$ & $89.8 \%$ \\
\hline $45^{\circ}$ & $-130^{\circ}$ & $\theta$ & $84.4 \%$ \\
\hline $45^{\circ}$ & $-130^{\circ}$ & $\varphi$ & $77.1 \%$ \\
\hline $45^{\circ}$ & $-45^{\circ}$ & $\theta$ & $84.5 \%$ \\
\hline $45^{\circ}$ & $-45^{\circ}$ & $\varphi$ & $71.8 \%$ \\
\hline $130^{\circ}$ & $0^{\circ}$ & $\theta$ & $77.6 \%$ \\
\hline $130^{\circ}$ & $0^{\circ}$ & $\varphi$ & $90.8 \%$ \\
\hline$-130^{\circ}$ & $0^{\circ}$ & $\theta$ & $68.1 \%$ \\
\hline$-130^{\circ}$ & $0^{\circ}$ & $\varphi$ & $92.0 \%$ \\
\hline$-45^{\circ}$ & $0^{\circ}$ & $\theta$ & $50.0 \%$ \\
\hline$-45^{\circ}$ & $0^{\circ}$ & $\varphi$ & $90.0 \%$ \\
\hline $45^{\circ}$ & $45^{\circ}$ & $\theta$ & $88.8 \%$ \\
\hline $45^{\circ}$ & $45^{\circ}$ & $\varphi$ & $98.0 \%$ \\
\hline
\end{tabular}

Figure 8 displays a comparison between the three worst results (in italics) and the best (98\%) and reference ones.
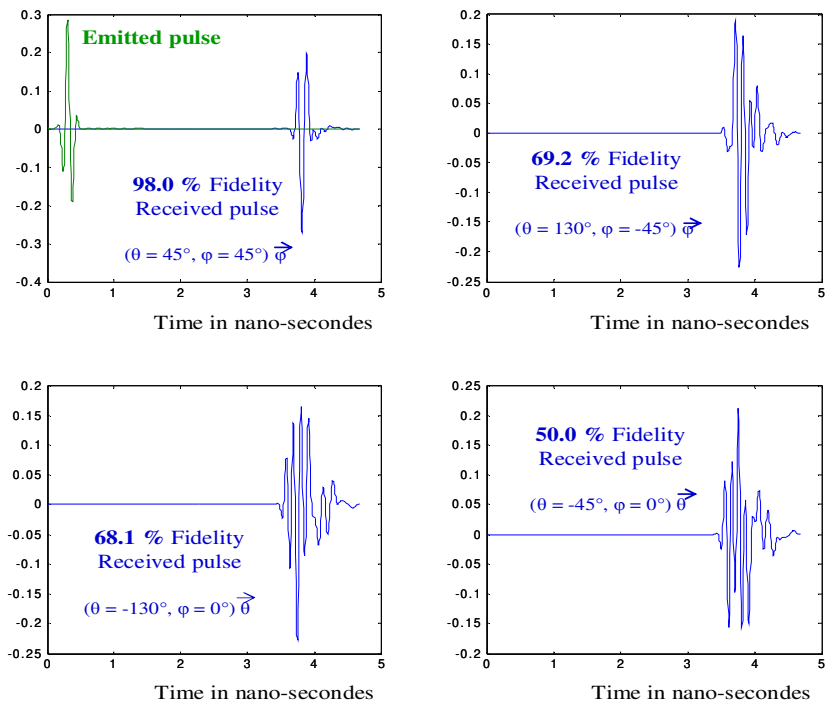

Figure 8: Normalized pulses at different angles $(\theta, \varphi)$. The three worst results (top right and lower raw) are compared to the best and reference ones (top left) in terms of fidelity.

At any location, it is possible to reach more than $83 \%$, depending upon the orientation. These results allow us to argue about the dispersion of the antenna: the latter seems to bee not so destructive, from the pulse shape point of view. The modification of the pulse by the radiation is not the same 
in different planes. Radiation pattern and matching performances give the opportunity to optimize the design in accordance with dispersion properties. Fidelity results confirm the potential of this antenna for UWB.

\section{IV - MEASUREMENTS OF THE ANTENNA}

The antenna was manufactured and measured. Due to the process tolerance and the small ground plane, the width of the impedance line and taper slightly varies and generate a shift in frequency, as seen in figure 9 . We thus reintroduce the variation in the simulator and the bandwidth retro-simulated is $5-12 \mathrm{GHz}$. The small size of the ground plane implies a high sensitivity of this antenna, as the figure 10 confirms this. This can be seen as a drawback but the reduction of the ground plane enables the possibility of isotropy characteristic. Increase of this ground plane would result in the reduction of the isotropy factor. This would be logically done for omnidirectional antenna design.

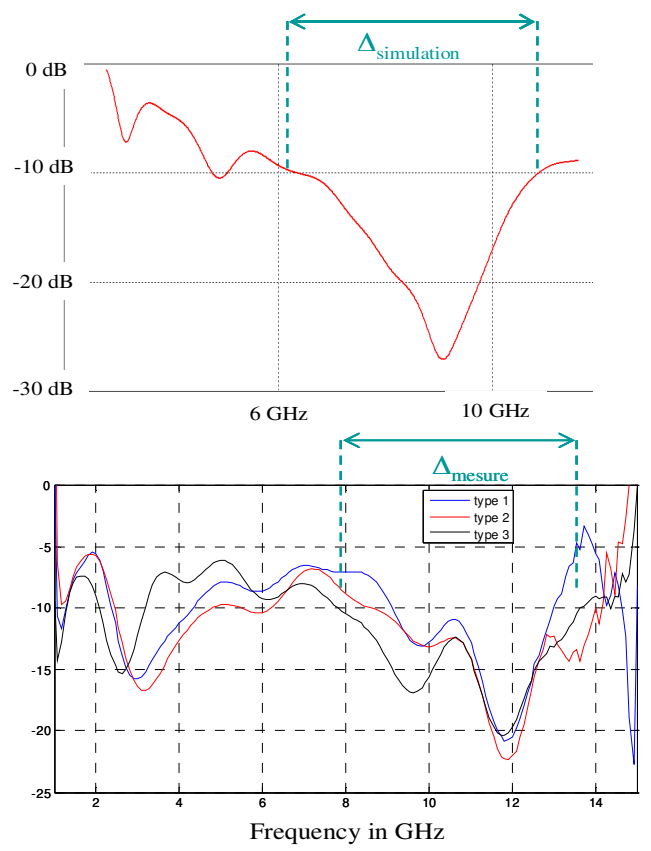

Figure 9: (retro-) Simulation (top) and $S_{11}$ measurements (bottom)

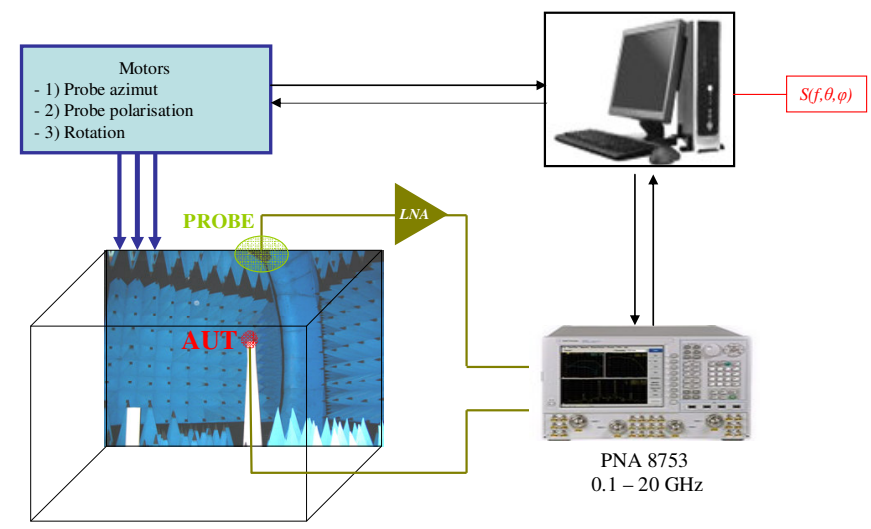

Figure 10: $S_{21}$ parameter measurements in SESAME
In Figure 9, type 2 and type 3 antennas correspond to improvements such as eccentricity enhancement $(+2 \%)$ for type 2 and addition of a coupled circular patch on the nonradiating face for type 3 . These modifications act as bandwidth enhancement due to the increase of the propagation surface, respecting the principle of traveling wave (printed) antennas.

Transmission measurements were done thanks to the spherical far-field test facility "SESAME" in Supelec (LSSDRE) as represented in figure 10. Due to the length of the cables (almost 10 meters), a LNA (0.2-18 GHz Mitech, $+30 \mathrm{~dB}$ medium gain, $+3 \mathrm{~dB}$ Noise Factor) was added to improve the measurement dynamic, unless the addition of noise. This is necessary because an isotropic antenna has gain in the range of $0 \mathrm{dBi}$. The PNA8753 used cannot evaluate the reflection coefficient of the system due to the system crippling losses at $\mathrm{GHz}$ frequencies. After calibrating the transmission with a reference probe, the $S_{21}$ parameter is extracted. Results of the RP are shown in figure 11. The gain varies from $+6 \mathrm{dBi}$ to $-20 \mathrm{dBi}$ in this figure. The directivity of the antenna is very sensitive to the ground plane due to the large radiating area. Figure 11 shows the quasi-isotropic and wideband behavior of the antenna because the top views are plotted at 10, 11 and $12 \mathrm{GHz}$.

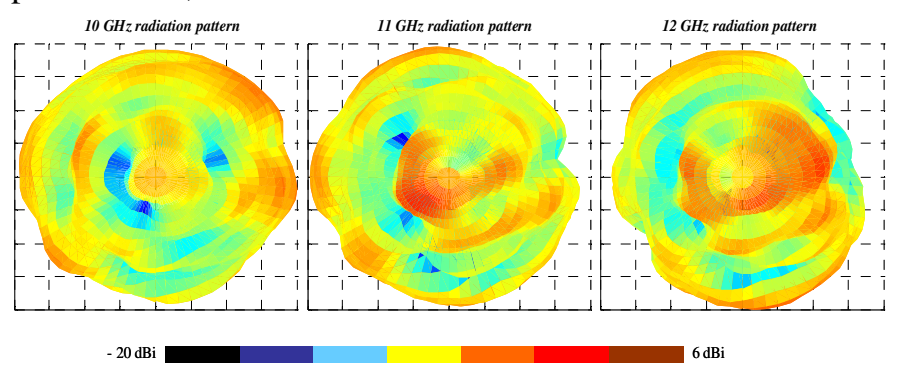

Figure 11: measured RP at 10, 11, and $12 \mathrm{GHz}$.

$S_{21}$ measurements are used to evaluate the efficiency of the antenna (de-embedding of the probe contribution). The efficiency is computed for the two polarizations, figure 12 . These results are in good agreement with the measured return loss of the antenna, figure 10.

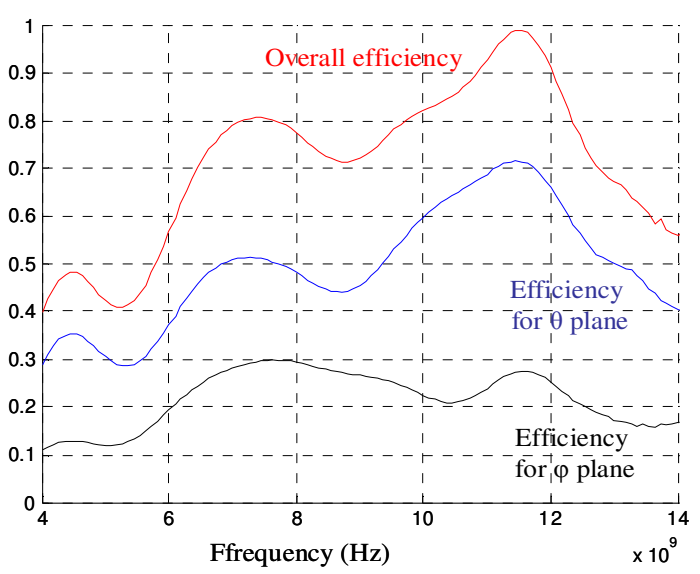

Figure 12: Efficiency thanks to the RP measurements. 
Transmitting and receiving UWB impulse signal implies the antenna $S_{21}$ parameter, in any direction $(\theta, \varphi)$, to be as invariant as possible in amplitude and phase over the largest bandwidth. Dispersion and compression would result in the destruction of the pulse shape and decrease the fidelity at a crippling level. Thanks to the measurement platform, we computed statistically the number of directions $\left(5^{\circ}\right.$ on $5^{\circ}$ solid angle step) for which $S_{21}(f, \theta, \varphi)$ has

- a minimum superior to $10 \mathrm{dBi}$

- a minimum to maximum level variation inferior to 10,13 and $16 \mathrm{~dB}$.

For different frequency bandwidths of interest: 3-10, 3-5, 4-6, 6-8, 6-12 and 8-13 GHz. Results are reported figure 13.
The variation reported in figure 13 reveals an amplitude distortion on the transmitted pulse. $S_{2 I}$ variations enable to identify the worse case of the antenna influence over a given bandwidth. A high sensitivity with the polarization in the $\theta$ plane is shown. This can be related to the non-symmetry of the antenna.

The phase variation is also de-embedded from measurements and reported in figure 14 for the two polarizations vectors. These results drive us to limit the maximum variation to \pm 10 degrees between 1 and $15 \mathrm{GHz}$. This measure is important for studying the worst case impact of such antennas in UWB impulse radio systems.
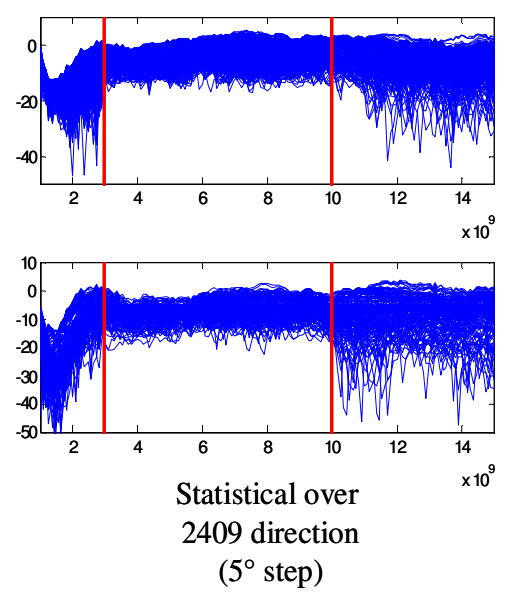

"3-10 GHz case (-10dB)"

\begin{tabular}{|c||c|c|c|c|c|c||}
\hline \multicolumn{1}{|c|}{} & \multicolumn{2}{|c|}{ MAX-MIN $<10 \mathrm{~dB}$} & \multicolumn{2}{c|}{ MAX-MIN $<13 \mathrm{~dB}$} & \multicolumn{2}{c|}{ MAX-MIN < 16 dB } \\
\cline { 2 - 7 } & $\begin{array}{c}\text { Polarisation } \\
\theta\end{array}$ & $\begin{array}{c}\text { Polarisation } \\
\varphi\end{array}$ & $\begin{array}{c}\text { Polarisation } \\
\theta\end{array}$ & $\begin{array}{c}\text { Polarisation } \\
\varphi\end{array}$ & $\begin{array}{c}\text { Polarisation } \\
\theta\end{array}$ & $\begin{array}{c}\text { Polarisation } \\
\varphi\end{array}$ \\
\hline $3-10 \mathrm{GHz}$ & $11 \%$ & $3 \%$ & $24 \%$ & $8 \%$ & $41 \%$ & $18 \%$ \\
\hline $3-5 \mathrm{GHz}$ & $54 \%$ & $41 \%$ & $72 \%$ & $57 \%$ & $83 \%$ & $70 \%$ \\
\hline $4-6 \mathrm{GHz}$ & $63 \%$ & $45 \%$ & $77 \%$ & $61 \%$ & $86 \%$ & $72 \%$ \\
\hline $6-8 \mathrm{GHz}$ & $56 \%$ & $38 \%$ & $70 \%$ & $53 \%$ & $78 \%$ & $67 \%$ \\
\hline $6-12 \mathrm{GHz}$ & $21 \%$ & $7 \%$ & $37 \%$ & $16 \%$ & $52 \%$ & $30 \%$ \\
\hline $8-13 \mathrm{GHz}$ & $34 \%$ & $14 \%$ & $54 \%$ & $30 \%$ & $66 \%$ & $43 \%$ \\
\hline
\end{tabular}

\begin{tabular}{|c|c|}
\hline \multicolumn{2}{|c|}{ MIN > -10 dB } \\
\hline $\begin{array}{c}\text { Polarisation } \\
\theta\end{array}$ & $\begin{array}{c}\text { Polarisation } \\
\varphi\end{array}$ \\
\hline $12 \%$ & $1 \%$ \\
\hline $27 \%$ & $13 \%$ \\
\hline $46 \%$ & $13 \%$ \\
\hline $47 \%$ & $21 \%$ \\
\hline $28 \%$ & $4 \%$ \\
\hline $37 \%$ & $8 \%$ \\
\hline
\end{tabular}

Figure 13: Statistical results for the amplitude variation in function of the direction

Phase dispersion (in degrees), polarization $\theta / \varphi$
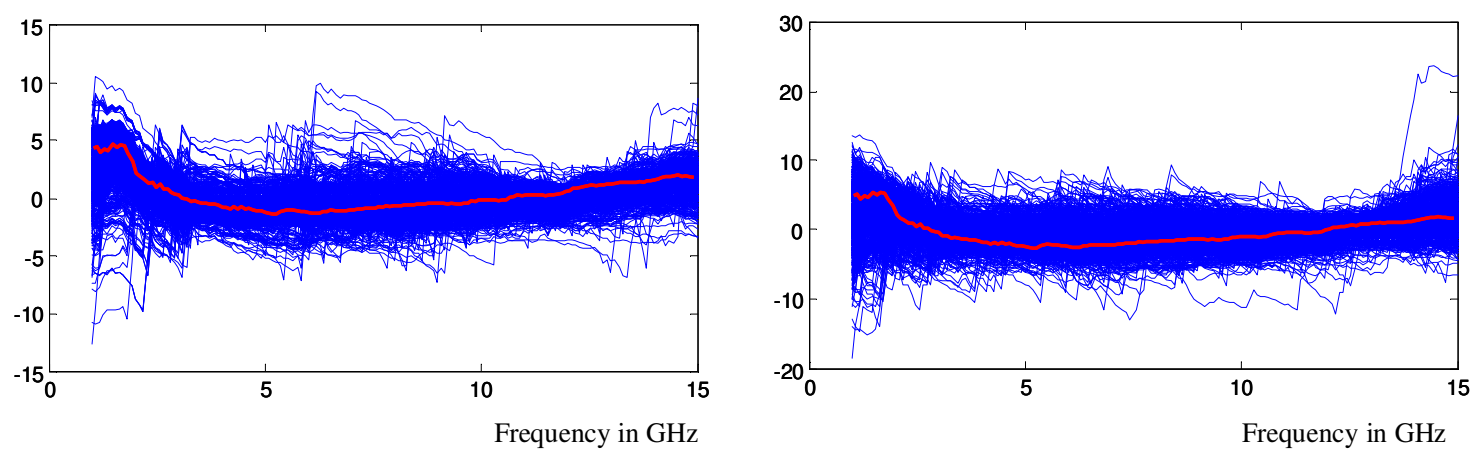

Figure 14: Phase response in degrees for the two polarisation vectors $(\theta$ on the left, and $\varphi$ on the right. Red curves is the de-embedded response of the LNA, connected to the reference horn antenna probe.

\section{Conclusion}

The antenna designed in this paper is based on the application of major principles on wideband (travelling waves) isotropic antennas. The adaptation from 3D volumetric to $2 \mathrm{D}$ printed structure is achieved as the comparison between simulations and measurements is satisfying. Using this type of antenna for impulse radio is possible as the fidelity is important and the amplitude and phase variations are in acceptable ranges.

As the antenna is designed for isotropy and not only omni-directivity, its RP is difficult to measures and its EM sensitivity is high. This is due to the weak difference between 
antenna gains in the different directions. $S_{21}$ coefficients are measured for a printed low cost prototype antenna and give some reference values for reintroducing the propagation channel in the performance evaluation of a wireless UWB communication system.

\section{REFERENCES}

[1] M. Ghavami, L. Michael and R. Khono. "UWB signals and systems in communications engineering" Wiley 2004

[2] H. Shantz. "A brief history of UWB antennas" Proceedings of IEEE UWB Systems and Technologies, 2003.

[3] H. Shantz. "Radiation efficiency of UWB antennas" Proceedings of IEEE UWB Systems and Technologies, 2002.

[4] R. Schmitt. "Understanding electromagnetic fields and antenna radiation takes (almost) no math" EDN mag., $N^{\circ} 3$ 2000, pp 77-88.

[5] M. A. Peyrot-Solis and al. "State of the Art in UWB Antennas" $2^{\text {nd }}$ International Conf. on Electrical and Electronics Engineering (ICEEE), Mexico, Sept. 2005.

[6] H. Schantz. "Dispersion and UWB Antennas" IEEE Antennas and Propagation Symposium'05, 2005.

[7] H. Schantz. "A near field propagation law and a novel limit to antenna gain Vs size" IEEE Antennas and Propagation Symposium, 2005.

[8] D. C. Cheng. "Update various kinds of UWB antennas" IEEE UWB Symposium 2005, Taïwan.

[9] T. Ma, S. Jeng. „Planar miniature tapered slot fed annular slot antennas for UWB radios" IEEE transactions on Antennas and Propagation, $N^{\circ} 3,2005$ pp 215-226.

[10] H. Schantz. "The Art and Science of UltraWideBand Antennas" Artech House, 2005.

[11] R. Scholtz and al. "Ultra-Wideband Radio" EURASIP Journal on Applied Signal Processing, March 2005, pp 252-272.

[12] V. Rumsey. "Frequency independent antenna" IRE National Convention Record, pt. I, 1957, pp 114-118.

[13] W. Sörgel et al. "Optimizing antenna performance for UWB impulse systems" EuCAP 2006.

[14] K. Chan et al. "A Novel CPS-fed Balanced Wideband dipole for UWB applications" EuCAP 2006.

[15] R. Serrano et al. "Small antenna fundamentals and technologies: future trends" EuCAP 2006. 